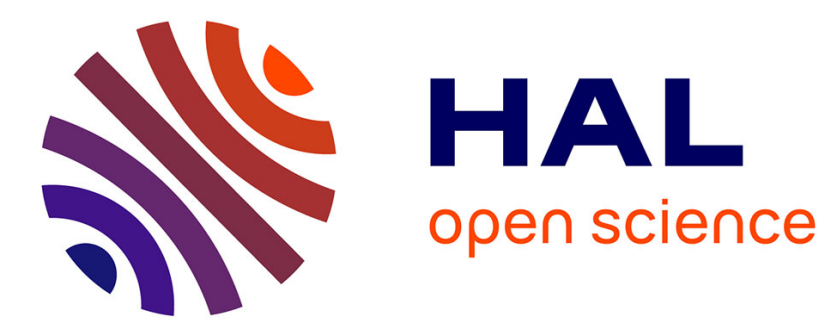

\title{
What is about future high speed power line communication systems for in-vehicles networks? \\ Fabienne Nouvel, Philippe Tanguy
}

\section{To cite this version:}

Fabienne Nouvel, Philippe Tanguy. What is about future high speed power line communication systems for in-vehicles networks?. Information, Communications and Signal Processing, 2009. ICICS 2009., Dec 2009, MACAU, China. pp.1-6. hal-00456386

\section{HAL Id: hal-00456386 https://hal.science/hal-00456386}

Submitted on 15 Feb 2010

HAL is a multi-disciplinary open access archive for the deposit and dissemination of scientific research documents, whether they are published or not. The documents may come from teaching and research institutions in France or abroad, or from public or private research centers.
L'archive ouverte pluridisciplinaire HAL, est destinée au dépôt et à la diffusion de documents scientifiques de niveau recherche, publiés ou non, émanant des établissements d'enseignement et de recherche français ou étrangers, des laboratoires publics ou privés. 


\section{What is about future high speed power line communication systems for in-vehicles networks?}

\author{
Fabienne Nouvel \\ IETR/INSA \\ Institut Electronique et Télécommunication de Rennes \\ Rennes, FRANCE \\ Fabienne.nouvel@insa-rennes.fr
}

\author{
Philippe Tanguy \\ IETR/INSA \\ Institut Electronique et Télécommunication de Rennes \\ Rennes, FRANCE \\ Philippe.tanguy1@insa-rennes.fr
}

\begin{abstract}
This paper deals with alternative automotive networks involved by the $\mathrm{X}$-by-wire and $\mathrm{X}$-tainment applications. New market demands like navigation, multimedia, security, safety and individualized options introduce more and more electronic control units. Furthermore, the automotive industry has to face a great challenge in its transition from mechanical engineering towards mechatronical products. In the last decades, the power line technology has received an increasing attention and spans several applications both in indoor, outdoor and invehicle data communications. To fulfil theses demands of intracar communications, techniques based on power line communication (PLC) seem to be a good candidate. These techniques offer both high data rate and good adequacy with power line properties. This paper revisits the work carried out in using PLC within the automotive domain. Different solutions are discussed and results are given for different applications.
\end{abstract}

Index Terms - vehicles, power line, X-by-wire, embedded networks.

\section{INTRODUCTION}

Nowadays, several fieldbus technologies, usually very specialized, were developed by car manufacturers to fulfil the different communications between electronic control units (ECU) according to their task. The ECU can assist the driver to control for example the vehicle through functions related to the steering, traction, braking system (ABS), Electronic Stability Program (ESP), Electric Power Steering (EPS), .... . The different used networks (i.e CAN, LIN, FlexRay, MOST) [1,chapter II and IV] provide different services. They required specific MAC and PHY layers for managing communications and, in particular, for granting bus access. Each of these networks use it own data lines and thereby is responsible for an increase of wiring harness. To reduce this complexity, without reducing safety, it is desirable to commit to a limited set of networks. However, it is not in the near future that the number of networks can be reduced to only one or two as such technology would provide the properties supporting the most demanding automotive systems. It would probably make it too expensive. Hence, it is more likely that a few network technologies will be used while providing timeliness and fault tolerance.

Among all the available technologies, it seems to be interesting to look at the evolution of the local are networks (LAN) for domestic uses. Since few years, power line communications (PLC) has received a great interest. PLC solution has numerous advantages, notably in terms of reliability, because it would reduce the amount of splicing that is necessary, as well as simplify and lighten the cable bundles The HomePlug Powerline Alliance [2] has been formed in 2000 by a group of industry leading companies to enable standards based powerline networking products. In 2005, the IEEE P1901 [3] has been created. The objectives of the IEEE P1901 working group are to standardize the PLC context. However, their efforts are limited to the physical layer (PHY) and medium access layer (MAC). Today PLC advantages have been demonstrated, it is the reason why the PLC solution is studied by car manufacturers. A first application of PLC could be redundant path for X-by-wire systems. Furthermore, PLC could be used for the diagnosis of ECUs and finally high data rate communications. In [4], the authors focus on PLC for automotive. The main issues proposed by the authors are the cost reduction, the bus segmentation and replacement of the common vehicle networks and especially the widespread CAN.

The remaining paper is organized as follows. In next section, we give an overview of previously work on high data rates PLC solutions. In section III, we discuss about automotive constraints. We briefly present the features of today PLC technologies used in indoor applications, namely the HomePlug $\mathrm{AV}$ and HD-PLC standards in section IV. In section $\mathrm{V}$, we present some results we obtain in our study. Finally, Section VI concludes the paper.

\section{BACK GROUND}

\section{A. Back ground: previous PLC studies in vehicle}

PLC is nowadays adopted in a multitude of application domains, both for broadband connectivity, indoor automation and data exchanges. A number of widely adopted communication protocols have been developed, starting from low speed X10 technology up to recent implementations like HomePlug or HD-PLC, which is also targeted to high speed communication, to support for example High Definition TV and VoIP. It is obvious that the power line in indoor has very few common with the automotive ones. However, the power line channels present numerous similarities: impulse noise, multipaths, attenuation [5] [6].

In [7], PLC feasibility for cars has been demonstrated. The proposed solution was based on spread spectrum technology. It 
allowed multi-user access by using code division multiple accesses. However, it achieved only a $50 \mathrm{Kbps}$ data rate per function. In [8], a CAN PLC transceiver has been studied. It supported both single carrier modulation and base band ASK modulation. It achieved bit rate up to $250 \mathrm{Kbps}$. Further investigations have been carried out in [9] in order to develop a CAN-based powerline multi-carrier transceiver instead of single carrier modulation. Today, the Yamar [10] Company proposed solution based on transmission over DC lines and CAN protocol. Two devices namely DCAN250 and DCB500 are already available. The DCAN250 allows communications up to $250 \mathrm{Kbps}$ as DCB500 allows various applications up to $500 \mathrm{Kbps}$. These PLC solutions have the advantages of being CAN compliant. However the main draw back is the low data rate and they do not answer X-by-wire and/or multi-media requirements.

\section{B. Recent PLC applications and investigations}

In more recent years, car manufacturers focus on PLC as an alternative for embedded networks due to its benefits related to the reduction of cabling and costs. In [11], authors investigate the properties of on board power lines and introduce a tutorial about the realization of high speed PLC. They propose a reorganization of the wiring harnesses based on a star structure. This solution can avoid reflection and makes impedance matching easier by using ferrite materials. Similar modifications of DC networks are proposed in [12] in order to fulfil high data rate requirements and safety. Recently, the PREDIT Project has focus on new embedded PLC solutions. In [5] the authors have studied high PLC data rate communication based on the well known OFDM technique (orthogonal frequency division multiple access). OFDM has the advantage to cope with the frequency selectivity of the power line channel caused by multiple reflections and by the coupling to cables. In the vehicle demonstration [12], a video transmission between a camera at the rear of the vehicle and a screen placed on the dashboard has been carried out up to 30 frames per second. Nowadays, one can affirm that the feasibility of indoor PLC over in-vehicle powerline channels is demonstrated and we need to go deeper into the study.

The workshop held in Italy [13] is aimed at presenting the state of the art in communication systems in a modern car, in order to optimize and reduce the cabling on board. Some useful results expected from the workshop are: a general layout of a useful benchmark; the specifications expected from a PLC based protocol to meet the automotive requirements; a list of significant experiments aimed at defining the viability of a PLC solution.

Recently, the author in [14] has proposed a vehicular communication system which can switch over a communication method between the orthogonal frequency division multiplex (OFDM) method and the spread spectrum (SS) method. A PLC master set includes a LAN communication unit for performing data communications with control units such as an engine control ECU via an in-vehicle LAN. In the PLC master set, a microcomputer acquires the operating states of parts of the vehicle from various control units through the LAN communication unit, and uses the acquired operating states and control information stored previously to enable data communications without being affected by noises. The demonstrator includes a camera that is mounted in a vehicle such as a car to photograph its periphery, and a receiving device that receives a video signal from the camera to perform image processing and the like are connected to enable data communications with each other through an electric power line wired in the vehicle and transmit the video signal from the camera to the receiving device through the power line. Furthermore in this system, the modulation per subcarrier can change from 64 QAM down to BPSK according to the channel state. However, no results in term of throughput are given nor bit error rates.

Among the different possible technologies that can be adopted in vehicle, a description of some of them is given in section IV.

\section{AutOMOTIVE POWER LINE AND CONSTRAINTS}

Before considering the PHY and MAC layers of PLC system, the capacity of the channel through the wires must be determined through channel measurements. Furthermore, the PLC solution must meet the EMC requirements.

\section{A. Channel measurement}

Different channel measurements have been carried out in [15] and [16] on different cars. The variations of the transfer function are observed in different configurations: electronic unit ON or OFF, ignition ON/OFF, car moving or not, battery connected or not. Additional complex paths (front, trunk, cockpit, point to point, multi-point ...) have been studied on an other car [17]. The throughput and channel measurement results show that it is not easy to confirm the separation into direct and indirect links as it has proposed in [18]. In [19], similar measurements have been performed in a small sports car. Experimentations show the effects of electronic states on the channel (drops of about $10 \mathrm{~dB}$ ), which are consistent with all the previous works. In addition, they do not confirm the direct and indirect path classification. Furthermore, the results emphasize that impedance adaptation for modem must be studied carefully as the interfaces will modify the power line channel. These different studies show that the channel characterization is not easy and linked to the vehicle.

Fig.1 and Fig.2 present two examples of variations of the transfer function between nodes in the vehicle. In these measurements, the motor is off. In Fig.1, D1D3 is considered as direct, as D1D2 and D2D3 are indirect as classified in [18]. All these points are in the Front area. We have done the measures in Fig.2 on a French Peugeot 407 SW. In most of the experimentations, the measurements are carried out up to 80-90 $\mathrm{MHz}$, as the upper bound for domestic channel is $100 \mathrm{MHz}$. 


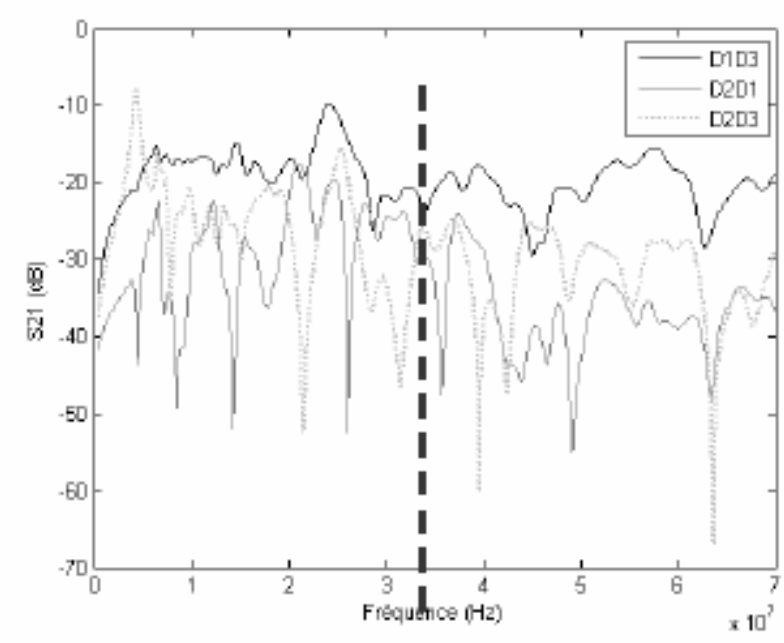

Figure 1. Channel measurement, short length Front - Front Area ( D1D3 : direct, D2-D1/D3 indirect), from [16]

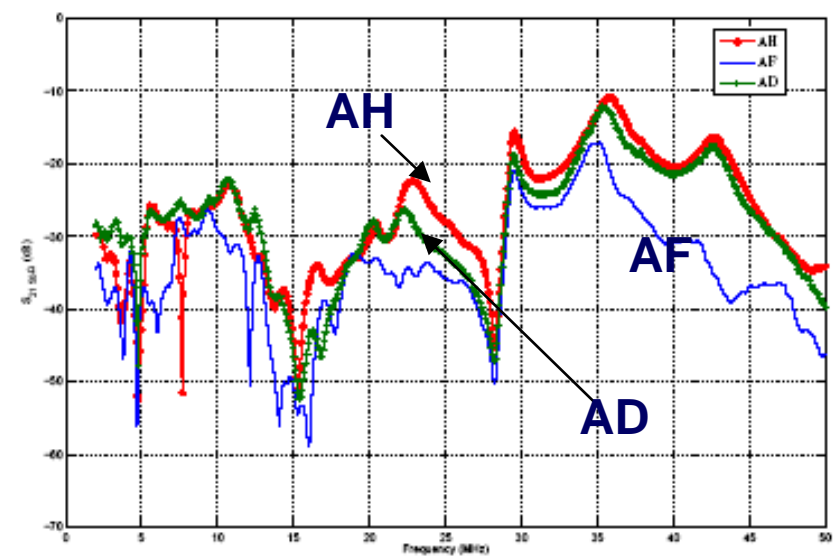

Figure 2. Channel measurement, between Front-Rear Area ( A- RearLeft, D- FrontLeft, F-FrontMiddle, H-FrontRight)

These results can be compared with indoor PLC profiles obtained in [20], represented in Fig.3. We can observe that this channel is not flat and is linked with the loads.

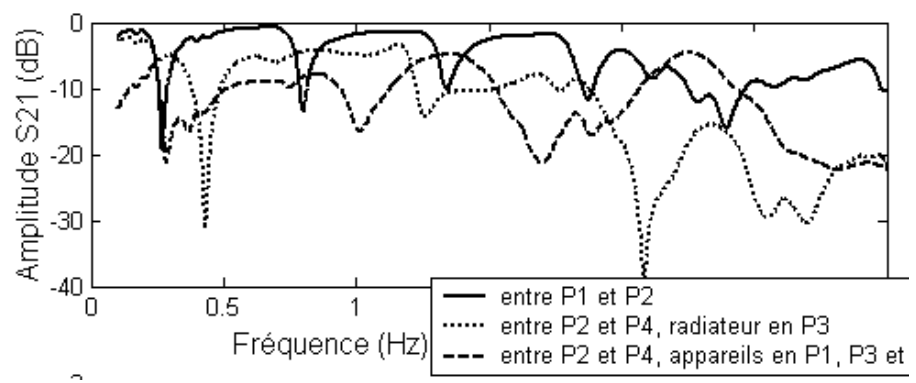

Figure 3. Indoor Channel measurement, from [20]- COST 2005, slide Number 14, bandwidth 0-30 MHz
In Fig.1 and Fig.2, we can observe the short length paths less suffer from reflection, cable attenuation and junction. The differences between are on the order of 10 up to $15 \mathrm{~dB}$ in the [0-30] MHz band. However, the D2D3 link presents deep fading due to the low battery impedance connected on this link. In the upper band, we can observe that for short links, the channels do not present high attenuation and could be an interesting transmission band. For the Front-Rear links (Fig.2), we observe a linear decrease. Results in [19] exhibit more reflections and variable insertion loss in the [30-100] MHz. However, those figures make it is clear that a narrow band and single carrier transmission is not able to provide efficient connectivity.

\section{B. EMC requirements}

The selected PHY will have to fulfil the PLC requirements defined in the Ray_CISPR25 [21] which allow transmission in the $[0.15-30] \mathrm{MHz}$ bandwidth under $45 \mathrm{~dB} \mu \mathrm{V}(-61 \mathrm{dBm})$ and $35 \mathrm{~dB} \mu \mathrm{V}(-72 \mathrm{dBm})$ for class 4 and class 5 respectively. Fig. 4 represents HomePLug AV power spectru measured on automotive power lines (the same car than Fig.2) compared with the EMC constraints. Letters correspond to different point in the vehicle, both Front Left/Right/Middle and Rear Left/Right/Middle. The access impedance has not been yet optimized. We can observe the PLC signal is CISPR compliant up to class 2.

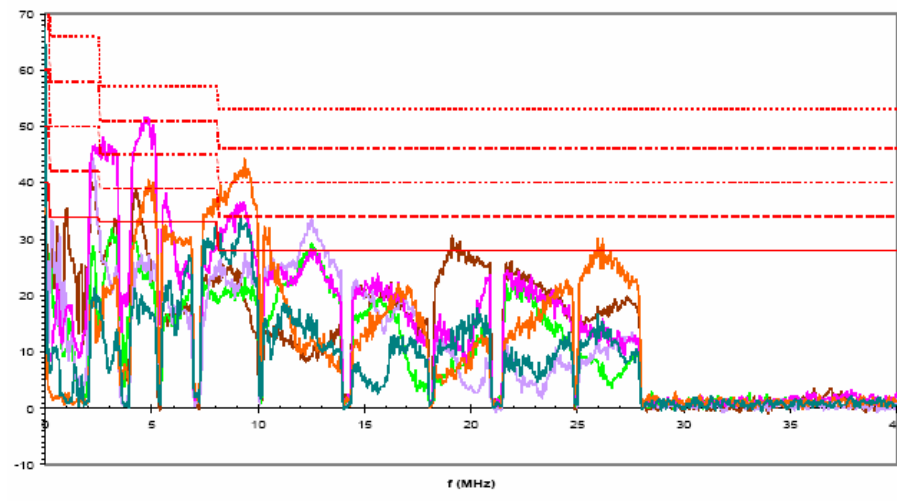

Figure 4. Automotive Power Spectrum HomePLug and CISPR requirements $(\mathrm{dB} \mu \mathrm{V})$

Those results can be compared with the previous ones obtained with other wide band PLC modems tested in cars [1, Chapter IV]. In this study, the access impedance has been optimized in order to both fulfil EMC constraints while achieving up to 15 Mbps data rate for different paths.

If we compare the CISPR vehicle requirements with PLC indoor's one, the indoor PLC emission mask may not exceed $50 \mathrm{dBm} / \mathrm{Hz}(57 \mathrm{~dB} \mu \mathrm{V})$ in North America. We notice this level is higher than the vehicular emission limit.

\section{PLC STANDARDS DESCRIPTION}

Considering these results, the PLC PHY layer will require frequency diversity which can be achieved by using orthogonal frequency division multiplex (OFDM). OFDM has been adopted for many wireless wide area or personal area networks (like in IEEE $802.11 \mathrm{a} / \mathrm{g}$ ) or over copper wires (ADSL). Actually, two standards are promoted by the IEEE 
1901 group, the HomePlug AV and the HD-PLC standard. The actual barrier to the adoption of PLC in indoor ( and then in car) is the lack of technical standard. We will rapidly described the two solutions proposed to standardization. However, additionnal systems have been proposed like the Spidcom technology.

\section{A. HomePLug 1.0 and HomePlug AV}

The HomePlug process is based on an OFDM technique whose major advantage for the embedded PLC application is to cope with the frequency selectivity of the power line channel. The HomePlug V1.0 [22] was based on 128(84 used) sub-carriers equally spaced from $4.3 \mathrm{MHz}$ to $25 \mathrm{MHz}$, (intercarrier space is about $195 \mathrm{KHz}$ ) with a $50 \mathrm{MHz}$ sampling rate. The OFDM symbol duration is $8.4 \mu$ s with $5.12 \mu$ s for the raw OFDM symbol and $3.28 \mu$ s for the guard interval (GI). The differential encoding allows simplifying the receiver part by suppressing the channel equalization. DBPSK, DQPSK or ROBO modulation on each subcarrier are applied according to the signal to noise ratio (SNR). Concerning the MAC layer, HomePlug V1.0 uses only the CSMA/CA protocol to access to the ad-hoc network. The enhanced HomePlug V1.0 is the HomePlug AV (HPAV) standard of which features are summarized in Table I. The OFDM modulation is realized thanks to a 3072 points IFFT/FFT with a frequency sampling of $75 \mathrm{MHz}$. In this case the OFDM symbol duration is 40.96 $\mu$ s and the space between each carrier is $24.414 \mathrm{kHz}$. The HPAV uses higher modulation order from BPSK (Binary phase-shift keying) up to 1024 QAM than HomePlug V1.0. Moreover, several GI can be used depending on the channel and so the data rates can be improved. A frequency mask is used to avoid interferences mainly with amateur radio bands. Thanks to this specific window, the out of band noise is reduced and the notches are deeper.

Those enhancements will improve many features: the throughput, the equalization, and the interferences. Results obtained in [18] show that the median values of coherence bandwidth (Bc) are $1.3 \mathrm{MHz}$ and $700 \mathrm{kHz}$ for direct and indirect connections. If we consider the delay spread (DS) and the GI, the authors report that the Ds remains under $380 \mu$ s for all the direct and indirect configurations. This delay is longer thanthe [2-5] $\mu$ s Ds obtained in indoor (it can also be on the order of some microseconds as in the cars). Generally, the GI duration should be about two to four times the DS.

If we compare the HPAV parameters with the $\mathrm{Bc}$ and Ds constraints, we can observe that they fulfil them. Furthermore, we can observe that the HPAV parameters are closer to the in vehicle channel parameters than the HomePlug V1.0 ones. These results are confirmed by the study presented in [15]. An enhanced OFDM system has been simulated using up to 2048 sub-carriers in the [0-20] MHz bandwidth. As the HomePLug V1.0 is not optimum to obtain a bit error rate (BER) of $10^{-3}$, this BER can be obtained by using a 1024 OFDM modulation with a SNR of $21 \mathrm{~dB}$. Similar study will be carried out in the actual study in order to improve the HPAV parameters for in vehicle PLC.
As the number of subcarriers increases, the throughput will be better and is given by:

$$
D=\frac{1}{\left(T_{s}+T_{g}\right)} \sum_{k=0}^{N_{p u}} m_{k} R
$$

where $m_{k}$ is the bits number per sub-carrier symbol defined according to the SNR, $\mathrm{R}$ the coding ratio, $\mathrm{N}_{\mathrm{pu}}$ the number of used subcarriers per OFDM symbol ( $\mathrm{N}_{\mathrm{p}}$ is the FFT size) and (Ts $+T_{g}$ ) the OFDM symbol duration. $T_{s}$ is related to the sampling frequency $F_{s}=N_{p} / T_{s}$, Tg is the guard interval. The ratio $\left(T_{s} /\left(T_{s}+T_{g}\right)\right)$ represents the loss due to the guard interval insertion. The increase of the GI will induce a decrease of the data rate and the spectral efficiency. On the contrary, we notice the HD-PLC does not use GI.

\section{B. $H D-P L C$}

The HD-PLC alliance [23] (HDPLCA) is an additional group which promotes the PLC networks based on its HD-PLC technology. It is based on a specific OFDM modulation called Wavelet-OFDM which exploits the Wavelet transform. It appears with this modulation that the notches are deeper than OFDM realized with IFFT/FFT. Moreover, Wavelet-OFDM does not use guard interval and so it has a better spectral efficiency than OFDM modulation. Pulse-Amplitude Modulation (PAM) with a order from 2 to 32 is used for modulation. The MAC layer uses the hybrid TDMA and CSMA/CA protocols synchronized with the AC line cycle. This solution seems to be very promising for vehicle PLC and may fulfil the vehicle channel requirements (coherence bandwidth for example).

These two standards are tested in the next Section and discussed.

\section{Spidcom}

Spidcom has built modems using OFDM and studied for car in [15]. The frequency range is [2 - 30] $\mathrm{MHz}$ with an original division in 7 independent sub-bands of 128 carriers each. A tone ordering is applied on each sub-carrier. Moreover, the FEC is a convolutional coder combined with a Reed Solomon coder. Such system can provide up to $10 \mathrm{Mbps}$ throughput (Front-Front path) taking into account the EMC requirements. However, they do not have been selected for standardization by the P1901 group. 
TABLE I. AVAILABLE PLC STANDARDS PARAMETERS

\begin{tabular}{|c|c|c|c|}
\hline Parameters & HPAV & Spidcom & HD-PLC \\
\hline Scrambler & yes & $\mathrm{NC}$ & $\mathrm{NC}$ \\
\hline FEC & Turbo code & $\begin{array}{l}\text { Convolutional, } \\
\text { Reed } \\
\text { Solomon } \\
\text { code }\end{array}$ & $\begin{array}{l}\text { LDPC; Con- } \\
\text { volutional, } \\
\text { Reed } \\
\text { Solomon } \\
\text { code }\end{array}$ \\
\hline Interleaver & yes & $\mathrm{NC}$ & $\mathrm{NC}$ \\
\hline Mapper & $\begin{array}{l}\text { QAM } \\
2-1024\end{array}$ & $\begin{array}{c}\text { BPSK-QAM } \\
256 \\
\end{array}$ & PAM 2-32 \\
\hline Modulation & $\begin{array}{l}\text { Windowed- } \\
\text { OFDM }\end{array}$ & OFDM & $\begin{array}{l}\text { Wavelet- } \\
\text { OFDM }\end{array}$ \\
\hline IFFT/FFT size & 3072 & 256 & - \\
\hline $\begin{array}{l}\text { Number of car- } \\
\text { rier }\end{array}$ & 1536 & $7 * 128$ & $\begin{array}{c}512 \\
\text { (extendable } \\
\text { to 2048) }\end{array}$ \\
\hline $\begin{array}{l}\text { Sampling } \\
\text { frequency }\end{array}$ & $75 \mathrm{MHz}$ & $\mathrm{NC}$ & $62.5 \mathrm{MHz}$ \\
\hline Bandwidth & $2-28 \mathrm{MHz}$ & $2-30 \mathrm{MHz}$ & $2-28 \mathrm{MHz}$ \\
\hline $\begin{array}{l}\text { Inter-carrier } \\
\text { space }\end{array}$ & $24.414 \mathrm{KHz}$ & $\mathrm{NC}$ & $122.07 \mathrm{KHz}$ \\
\hline Symbol duration & $40.96 \mu s$ & $\mathrm{NC}$ & $8.192 \mu \mathrm{s}$ \\
\hline $\begin{array}{l}\text { Guard Interval } \\
\text { duration }\end{array}$ & $\begin{array}{c}5.56 \mu s \text { or } \\
7.56 \mu s \text { or } \\
47.12 \mu s\end{array}$ & $\mathrm{NC}$ & - \\
\hline $\begin{array}{l}\text { MAC layer pro- } \\
\text { tocol }\end{array}$ & $\begin{array}{l}\text { Hybrid: } \\
\text { CSMA/CA } \\
\text { \& TDMA }\end{array}$ & $\begin{array}{l}\text { TDMA and } \\
\text { CSMA/CA }\end{array}$ & $\begin{array}{l}\text { Hybrid: } \\
\text { CSMA/CA } \\
\text { \& TDMA }\end{array}$ \\
\hline
\end{tabular}

\section{PLC OVER VEHICLE DC CHANNELS}

In this section, we will give some comparison between the two main standards, namely HPAV and HD-PLC. We will focus on some perspectives.

\section{A. HomePlug AV and HD-PLC spectrum}

We have measured the two spectrums over the DC channels according to the configuration presented in [24]. Fig. 5 illustrates these two spectrums.

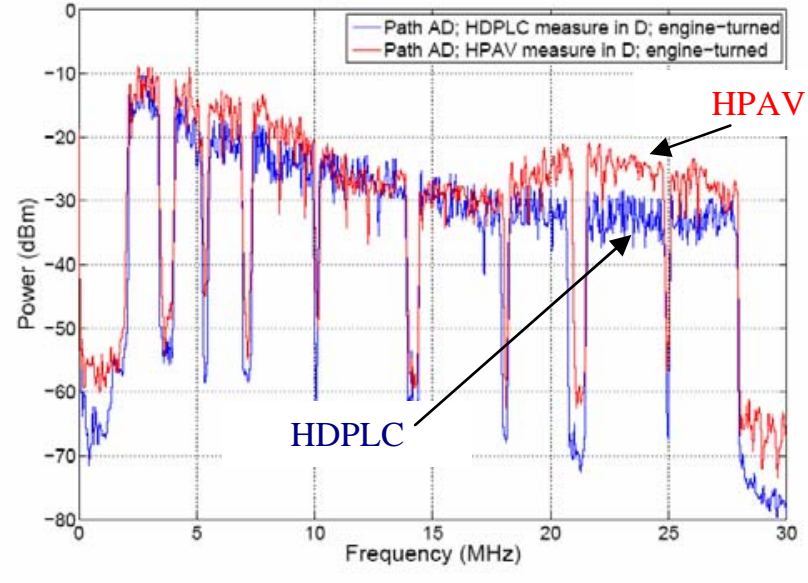

Figure 5. HD-PLC versus HPAV over vehicular power lines

The input impedance has not been yet modified and the transmitted OFDM waveform is not EMC compliant. We can observe that HD-PLC presents deeper notches than HPAV with a less emission power. The measurements have been performed with the same configuration (e.g. similar path, equipments $\mathrm{OFF}$, motor $\mathrm{ON}$ ); however the impedance of the modems may be different. It is not possible to use the two protocols at the same time as they have non-interoperable PHY layers.

\section{B. HomePlug AV and HD-PLC throughput}

The throughput has been measured using UDP traffic. For Front-Rear paths, we obtain about $15 \mathrm{Mbps} / \mathrm{s}$ for both systems. For Front-Front paths, the mean data rate is about 15 up to 25 Mbps. The highest one is 40 Mbps using the shortest FronFront path. We have noticed very low variations when the electronic units are in active use and the engine is driven. These results are better than the previous ones obtained in [15] [16]. In fact, the enhanced OFDM HPAV loads each tone according to the SNR for each connection between transmitter and receiver (e.g., modulation per tone and cyclic prefix length). Furthermore, the coding schemes (Turbo or LDPC) have better performances than RS or convolutional codes used in HomePlug V1.0.

However, even these results present great challenges for PLC in automotive, we need to focus on different technical requirements and next steps.

\section{Discussion about PLC solutions.}

1) Static notches: We have seen that notches are required to avoid interferences with amateur bands. Such notches are not necessary in vehicle environment. On the contrar, we have to take care with the upper FM band (80-100 MHz) and EMC constraints. Notches may be created dynamically through configuration according to the channel environment (detection of narrow radio band for example).

2) EMC requirements: The level of the transmitted signal need to be reduced and will induce a loss of throughput. Howver, the transmission bandwidth can be extended up to 50 $\mathrm{MHz}$ as no overall drops occurs. 
3) Synchronisation between nodes: in the two systems, the zero crossing of the AC line is used to synchronize the nodes with a master node which generates a beacon period. This beacon contains information about the contention free and carier sense multiple access (CSMA/CA) region. In vehicle, such synchronization is not possible. However, different states of the car can be detected to initialize the first beacon : ignition switch, battery state, ...

4) Mac layer: The two networks use a master-slave architecture. This master (chosen or fixe) authenticates the nodes and may assignes dynamic or static slots. For vehicle application, it seems to be better to identify a static master, always in active use. Additionnal PLC nodes will be considered as slaves.

\section{CONCLUSION}

The adaptation of PLC to vehicles holds great promises. Different experimentations have been presented. It is possible to achieve high throughput suited for different applications (multi-media, safety). Investigations of EMC disturbances have been pointed. Different adaptations of indoor PLC are proposed in order to fulfil vehicle constraints. They cope both with the MAC and PHY layers.

\section{ACKNOWLEDGMENT}

This work is carried out in the CIFAER project, initiated and supported by the ANR and the French Premium Cars Competitiveness Cluster.

\section{REFERENCES}

[1] Huaqun Guo. Automotive Informatics and Communicative Systems : Principles in vehicular networks and Data exchange. Chapter Automotive Network Architecture for ECU communication Information Science Reference, ISBN 978-60566-338_8,2009.

[2] Official website of the HomePlug Powerline Alliance (HPA). HomePlug Powerline Alliance. [Online]. Available: http://www.homeplug.org

[3] The IEEE P1901 draft standard for broadband over power line networks: Medium Access Control and Physical Layer Specifications. IEEE Standards Association. [Online]. Available: http://grouper.ieee.org/groups/1901/

[4] F. Benzi, T. Facchinetti, T. Nolte, and L. Almeida, "Towards the powerline alternative in automotive applications," in Factory Communication Systems, 2008. WFCS 2008. IEEE International Workshop on. IEEE Industrial Electronics Society, May 2008, pp. 259262.

[5] Lienard, M., Carrion, M., Degardin, V. \& Degauque, P. Modeling and Analysis on In-vehicle power line communication channels, IEEE Transaction Vehicular Technologie, vol 57, №2, (pp 670-679), 2008

[6] Degardin, V , Rouissi F., Lienard, Degauque P. \& Zeddam A., Comparison of in-house and in-vehicle noise characteristics in PLC systems, URSI 2008, Available : http:// ursitest.intec.ugent.be/files/URSIGA08/papers/E07p2.pdf
[7] F. Nouvel, G. El Zein, and J. Citerne, "Code division multiple access for an automotive area network over power-lines," in Vehicular Technology Conference, 1994 IEEE 44th, June 1994, pp. 525-529 vol.1.

[8] H. Beikirch and M. Voss, "Can-transceiver for field bus powerline communications," in Power Line Communications and Its Applications, 2000 IEEE International Symposium on, 2000, pp. 257-264.

[9] J. Taub, H. Beikirch, and M. Voss, "Real-time capabilities with digital powerline communications interfaces in csma/ca-networks," in Proc. Of RTN 2004, 3rd Int. Workshop on Real-Time Networks, 2004.

[10] Official web site of Yamar. Yamar Electronics LTD. [Online]. Available: http://www.yamar.com

[11] T. Huck, J. Schirmer, T. Hogenmuller, and K. Dostert, "Tutorial about the implementation of a vehicular high speed communication system," in Power Line Communications and Its Applications, 2005 International Symposium on, pp. 162-166April 2005.

[12] Electrical and electronic distribution systems: Focus on power line communication. Valeo Inc. [Online]. Available: http://www.valeo.com

[13] WORKSHOP Fieldbuses for automotive and the powerline alternative Università degli Studi di Pavia, 24-25 January 2008, Italy

[14] T. Mizutani \&D. Corporation, "Vehicular communication device and control information generating device“, Patent, IPC8: AH04J1100F1, Available on line http://www.faqs.org/patents/app/20080298226, 2008

[15] W. Gouret, F. Nouvel, and G. El Zein, "High data rate network using automotive powerline communication," in Proc. (IEEE) International Conference on Intelligent Transport System Telecommunications, pp. 14, June 2007.

[16] M. Carrion, M. Lienard, and V. Degauque, "Communication over vehicular Cc lines : propagation channel characteristics,” in Proc. (IEEE) International Conference Intelligent Transport System Telecommunications ITST2006, pp 2-5, 2006.

[17] Nouvel, F., Maziéro P., "X-by-wire and Intra-car communications: power line and/or wireless solutions". IEEE International ITST Conference, ITST 2008, pp 1-4, 2008

[18] M. Lienard, M. Carrion, V. Degardin, and V. Degauque, "Modeling and analysis of in-vehicle power line communication channels," IEEE Trans. Veh. Technol., vol. 57, no. 2, pp. 670-679, Mar. 2008.

[19] M. Mohammadi, L. Lampe, M. Lok, S. Mirabbasi, M. Mirvakili, R. Rosales, and P. van Veen, " Measurement Study and Transmission for In vehicle“, in International Symposium on Power Line Communications and Its Applications, ISPLC2009, Session 2A March 2009

[20] V. Degardin, P. Laly, M. Carrion, M. Liénard and Pierre Degauque, "Power Line Communication: Application to Indoor and In-Vehicle Data Transmission“, COST 286, 12-13 /12 2005, 2005

[21] Limits and methods of measurement of radio disturbance characteristics for the protection of receivers used on board vehicles. Technical report, CISPR-25, released 2002, relaesed 2008.

[22] M.K.Lee, R.E. Newman, H.A. Latcman, S. Katar and L. Yonge, “ HomPlug 1.0 PowerLine Communication LANs-Protocol”, International Journal of Communication Systems, 2000, Revised 18/09/2002, V1.01.

[23] Official website of the High Definition Power Line Communication Alliance (HDPLCA). HD-PLC Alliance. [Online]. Available: http://www.hd-plc.org/

[24] P. Tanguy, F. Nouvel, "Implementation of vehicular high speed power line communication system”. Submitted to IEEE International Globecom 2009, Honolulu, 2009 